\title{
ÁREAS ÚMIDAS BRASILEIRAS: BASES PARA O GERENCIAMENTO, SERVIÇOS ECOSSISTÊMICOS E ESTRATÉGIAS DE MANEJO
}

Fabio Leandro da Silva

Doutorando no Programa de Pós-Graduação em Ecologia e Recursos Naturais (PPGERN), Universidade Federal de São Carlos (UFSCar), São Carlos, SP, Brasil fabioleodasilva@gmail.com

Welber Senteio Smith

Professor titular da Universidade Paulista (UNIP), Campus Sorocaba, Laboratório de Ecologia Estrutural e Funcional de Ecossistemas. Professor do Programa de Pós-Graduação em Ciências da Engenharia Ambiental, Centro de Recursos Hídricos e Estudos Ambientais/CRHEA, Universidade de São Paulo, São Calos, SP, Brasil. welber smith@uol.com.br

Marcela Bianchessi da Cunha-Santino Professora Associada do Departamento de Hidrobiologia (DHb) da UFSCar. Professora e Orientadora do PPGERN/UFSCar, Campus de São Carlos, São Carlos, SP, Brasil cunha santino@ufscar.br

Irineu Bianchini Junior Professor Titular do DHb da UFSCar. Professor e Orientador do Programa do PPGERN/UFSCar, Campus de São Carlos, São Carlos, SP, Brasil irineu@ufscar.br

\begin{abstract}
RESUMO
O presente estudo buscou discutir o arcabouço legal relacionado com o gerenciamento de áreas úmidas no contexto brasileiro, apresentando os principais serviços ecossistêmicos prestados por esses sistemas aquáticos e a sua importância para a sociedade, além de apresentar estratégias de manejo pautadas na Ecologia Aplicada, visando alcançar a proteção da biodiversidade frente ao cenário de mudanças climáticas. As áreas úmidas são sistemas importantes, tanto do ponto de vista ambiental e socioeconômico, responsáveis por desempenham uma série de funções ecológicas (e.g. ciclagem de nutrientes, provisão de habitat para a biota, filtragem da água) e auxiliarem na subsistência e manutenção do ser humano. Os ecossistemas de áreas úmidas brasileiros carecem de um dispositivo normativo nacional que trate sobre a sua proteção. Dentre as principais estratégias, sobressaem-se: (i) a implementação de medidas com caráter estratégico e que integrem o planejamento de curto a longo prazo; (ii) a consideração dos componentes da paisagem e provisão de serviços ecossistêmicos e (iii) o monitoramento desses sistemas mediante a adoção de medidas que propiciem a manutenção da biodiversidade.
\end{abstract}

Palavras-chave: Ecossistemas aquáticos. Ecologia Aplicada. Estratégias.

\section{BRAZILIAN WETLANDS: BASES FOR MANAGEMENT, ECOSYSTEM SERVICES AND MANAGEMENT STRATEGIES}

\begin{abstract}
The present study aimed to discuss the conceptual framework related to the wetlands management in the Brazilian context, to discuss the main ecosystem services provided by these aquatic systems and their importance to society, as well as to present management strategies in applied ecology, in order to achieve the protection of biodiversity against the climate change scenario. Wetlands are important systems, both from an environmental and socioeconomic point of view, responsible for many ecological functions (e.g. nutrient cycling, provision of habitat for biota, water filtration) and assist in the subsistence and maintenance of humankind. Brazilian wetland ecosystems lack a national normative device that deals with their protection. Among the main strategies, the following stand out: (i) the implementation of measures with a strategic character and that integrate short to long term planning; (ii) the consideration of landscape components and the provision of ecosystem services and; (iii) the monitoring of these systems through the adoption of measures that favor the maintenance of biodiversity.
\end{abstract}

Keywords: Aquatic ecosystems. Applied Ecology. Strategies.

$\begin{array}{lllll}\text { Caminhos de Geografia } \quad \text { Uberlândia-MG } & \text { v. 22, n. } 79 & \text { Fev/2021 } & \text { p. 97-111 } & \text { Página } 97\end{array}$




\section{INTRODUÇÃO}

Os ecossistemas de áreas úmidas possuem uma forte e complexa associação com o regime hidrológico e participam de vários processos ecológicos, além de apresentarem propriedades específicas (e.g. edáficas, vegetação, fauna) e exercerem uma importante função como filtros naturais. Tais ecossistemas são considerados como uma área de transição entre o meio terrestre e aquático (TUNDISI e MATSUMURATUNDISI, 2010), podem ser classificadas como continentais ou costeiras, permanente ou periodicamente inundadas e possuem o solo constantemente alagados (JUNK et al., 2014). Esses corpos hídricos podem ser considerados ecossistemas estratégicos, visto que contribuem para a promoção da segurança alimentar, reduzem a ocorrência de desastres (e.g. enchentes e inundações) e auxiliam na proteção ambiental (VÉLEZ; GARCÍA; TENORIO, 2018).

As áreas úmidas podem desempenhar um importante papel no cenário de mudanças climáticas, visto que podem auxiliar na retenção dos Gases de Efeito Estufa (GEE). Evidências apontam que os ecossistemas de áreas úmidas contribuem para a produção de GEE (e.g. $\mathrm{CO}_{2}, \mathrm{~N}_{2} \mathrm{O}$ ), porém, apesar de serem uma importante fonte de emissão de metano $\left(\mathrm{CH}_{4}\right)$ em alguns casos, esses ecossistemas são também considerados importantes sumidouros de $\mathrm{CO}_{2}$ devido ao acúmulo de matéria orgânica (LIPCZYNSKIKOCHANY, 2018; SUCHARA, 2018).

Mesmo com toda a sua importância para a sociedade, as áreas úmidas têm sofrido rupturas em suas conexões com os ambientes lóticos e reduções em suas áreas, devido a sua conversão em outros usos da terra (e.g. áreas urbanizadas, áreas agrícolas), principalmente pela prevalência dos interesses ligados ao agronegócio (IORIS, 2013; WATSON et al., 2016). A análise da paisagem permite verificar os efeitos ecológicos sobre as áreas úmidas e quais os principais processos envolvidos na degradação ambiental (SILVA et al., 2020a). Yang et al. (2018) reforçam que a poluição da água e a invasão de espécies exóticas também se encontram entre as principais ameaças aos ecossistemas de áreas úmidas, assim como o desenvolvimento das atividades humanas. No Brasil, o cenário não é diferente, algumas medidas de caráter governamental têm contribuído para o agravamento da situação degradativa (e.g. Lei de Proteção da Vegetação Nativa, expansão do setor agrícola) e vão contra acordos firmados em nível internacional (e.g. Convenção Ramsar, Metas de Aichi), ameaçando a biodiversidade.

O processo de degradação que vem ocorrendo demanda a adoção de medidas que possam contribuir para a amenização ou até mesmo reversão desse processo, assim como o cumprimento das normas e acordos existentes. Omernik e Griffith (2014) destacam que os ecossistemas são extremamente complexos para o nosso entendimento, sendo assim, é preciso formular medidas que sejam úteis para a tomada de decisão. Nesse contexto, as áreas úmidas são ecossistemas complexos, situação esta que torna necessária a realização de uma avaliação desses ambientes e a consideração de elementos indispensáveis para o seu adequado gerenciamento, bem como a formulação de políticas para a sua proteção. Sendo assim, os governos locais assumem um importante papel (SMITH et al., 2018), uma vez que, podem desenvolver uma série de ações que propiciem mudanças no curso de degradação dos ecossistemas de áreas úmidas, situação que pode ser favorecida através do instrumento de planejamento territorial denominado Plano Diretor (SILVA et al., 2019a).

Dentro desse cenário e considerando o território brasileiro, surge a necessidade da adoção de medidas de manejo, contudo, primeiramente é necessária a obtenção de um conhecimento prévio sobre esses sistemas, a consideração da influência regional e o aparato normativo existente. Frente ao exposto, o presente trabalho buscou abordar: (i) elementos a serem considerados na formulação de políticas; (ii) o arcabouço legal relacionado com o gerenciamento de áreas úmidas no contexto brasileiro; (iii) os principais serviços ecossistêmicos prestados por esses sistemas aquáticos e a sua importância para a sociedade e (iv) estratégias de manejo pautadas na Ecologia Aplicada, visando alcançar a proteção da biodiversidade frente ao cenário de mudanças climáticas.

Para a realização da pesquisa, foi feita uma análise reflexiva pautado em referências disponíveis em bases de busca (Web of Science, Scopus) e bases oficiais sem filtro de tempo, de forma a obter informações acerca da temática abordada. 


\section{ELEMENTOS QUE DEVEM SER CONSIDERADOS NA ELABORAÇÃO DE POLÍTICAS VOLTADAS PARA AS ÁREAS ÚMIDAS BRASILEIRAS}

O que devem ser considerados na elaboração de políticas voltadas para as áreas úmidas brasileiras? A grande maioria das áreas úmidas brasileiras são moduladas pelo pulso de inundação, que varia conforme vários fatores (e.g. frequência, profundidade), entretanto, esses importantes elementos não costumam ser incorporados pelos planos voltados para o gerenciamento dos recursos hídricos e sofrem constantemente com os impactos do crescimento populacional e a poluição advinda das atividades humanas (JUNK, 2013).

Muitas populações tradicionais e famílias possuem uma relação direta com os ecossistemas de áreas úmidas, haja visto a retirada de seu sustento, assim como o seu conhecimento e esforços despendidos contribuem para a conservação e manejo destes sistemas (DIEGUES, 1991; PIYAPONG et al., 2019). Algumas populações possuem relações de religiosidade e espiritualidade com estes sistemas, como é o caso dos pantaneiros (GONÇALVES et al., 2018).

As necessidades da sociedade e elementos essenciais à manutenção dos sistemas naturais devem ser consideradas pela tomada de decisão do poder público, sociedade civil e terceiro setor, como também a relação de interdependência entre água e funcionamento dos ecossistemas e a relação com a ecohidrologia (XAVIER et al., 2019), subdisciplina da hidrologia que destina seus esforços nos processos ecológicos relacionados com o ciclo hidrológico, de modo a fazer uso destes processos sistêmicos e propriedades dos ecossistemas na promoção da sustentabilidade ambiental (ZALEWSKI, 2010).

Se faz necessário a realização de inventários e monitoramento ambiental, possibilitando um acompanhamento de eventuais perdas de áreas e interferências humanas, principalmente aquelas inseridas em áreas remotas e ameaçadas pelo agronegócio e crescimento urbano, bem como é preciso a elaboração de planos de recuperação dos ambientes já degradados através de uma estrutura proveniente da esfera governamental (KINGSFORD; BASSET; JACKSON, 2016). A influência da sazonalidade e o caráter temporário de algumas áreas úmidas pode dificultar o seu mapeamento através de técnicas de geoprocessamento e sensoriamento remoto (BOZELLI et al., 2019). Ainda segundo esses autores, mudanças acerca da visão de que estes ambientes inseridos em áreas urbanizadas tratam-se de "espaços vazios" dos ecossistemas terrestres e a não consideração e/ou reconhecimento das áreas sujeitas à conversão devido ao caráter temporário são elementos necessários a serem incorporados em políticas futuras, já que uma grande variação do nível de água.

Outro aspecto de destaque remete ao fato da necessidade de se preservar o fluxo genético das espécies que ocorrem nestes ecossistemas e a adoção de medidas que almejem conservar as populações em nível regional (CORRÊA et al., 2016). É imprescindível esforços para a preservação destes ecossistemas que apresentam condições pristinas, o que torna necessário o monitoramento ambiental, em especial nas localidades onde ocorre o desenvolvimento de atividades ligadas ao turismo (POLAZ; FERREIRA; PETRERE JÚNIOR., 2017). A conservação da biodiversidade, proteção do pescado, o combate a espécies exóticas/invasoras, serviços ecossistêmicos e critérios padronizados para a avaliação e o monitoramento das áreas úmidas são elementos necessários para a manutenção da integridade ecológica das áreas úmidas (CHEN; SHIH, 2019).

A lacuna acerca de definição e classificação das áreas úmidas está presente no aparato normativo brasileiro, da mesma forma que as peculiaridades hidrológicas destes sistemas ao longo do território nacional e os macrohabitats existentes não estão presentes em mecanismos normativos (JUNK; PIEDADE, 2015), situação que deveria ocorrer. Outrossim, a situação é agravada devido as lacunas existentes na Lei de Proteção da Vegetação Nativa - LPVN (Lei Federal 12.651/12), decorrente da redução e/ou não obrigatoriedade de manutenção das faixas de vegetação nativa a serem preservadas ao redor dos corpos hídricos e a antropização de áreas previamente protegidas (e.g. JUNK e PIEDADE, 2015; GRASEL et al., 2018; SILVA et al., 2019a; SILVA et al., 2019b). As áreas úmidas brasileiras menores que 01 hectare estão vulneráveis devido a redação da LPVN, que desobriga a manutenção de áreas de vegetação nativa ao redor desses ambientes (GRASEL et al., 2018). 
Uma postura que antecipe os impactos advindos da rápida mudança econômica e socioecológica é necessária para lidar com os efeitos do aquecimento global (JUNK, 2013). É preciso se considerar o potencial uso destes ecossistemas para a produção de biocombustíveis, o mercado de carbono, as emissões naturais de GEE, o papel destes sistemas no aquecimento global, ameaças à manutenção da qualidade da água e a mitigação de efeitos adversos provenientes de projetos relacionados com o desenvolvimento (BOSSELMAN, 2009). Se bem utilizada, as estruturas e ferramentas elaboradas pelo governo brasileiro podem auxiliar na redução da conversão de ecossistemas naturais (contribuindo na manutenção das áreas úmidas), como a consolidação do Cadastro Ambiental Rural auxilia (ROITMAN et al., 2018). Entretanto, é preciso a verificação in loco das informações fornecidas pelos proprietários rurais pelos órgãos ambientais e a correção de eventuais distorções.

Variações também podem ser observadas nos processos de decomposição, produtividade primária, estoque de carbono e provisão de biomassa (JUNK et al., 2014). A instituição de um ato ou dispositivo normativo que verse especialmente sobre as áreas úmidas podem favorecer a sua conservação, manejo e uso racional. Um exemplo, é a conotação legal e o ato de conservação das áreas úmidas instituídos por Taiwan na década passada, que busca a conservação e sustentabilidade destes ecossistemas desde o nível nacional até o local.

Diante de toda a complexidade acerca das áreas úmidas e todas as funções ambientais envolvidas (GOMES; MAGALHÃES JÚNIOR., 2017), a adoção de uma classificação se torna necessária.

\section{MECANISMOS NORMATIVOS}

Um dos principais marcos em relação aos corpos hídricos brasileiros é o Código de Águas (Decreto $n^{\circ}$ 24.643/1934) (BRASIL, 1934). Este dispositivo enquadra os ambientes de águas paradas e áreas pantanosas como locais de uso comum e indica que nos casos de insalubridade esses ambientes podem ser drenados. O decreto, porém, carece de critérios no que se refere à insalubridade e não aborda de maneira explícita os ecossistemas de áreas úmidas.

Ainda em relação ao meio ambiente e dispositivos que tratem da matéria ambiental, sobressai-se a Política Nacional de Meio Ambiente - PNMA (Lei Federal $n^{\circ}$ 6.938/1981) (BRASIL, 1981). Um aspecto importante imposto pela PNMA é o estabelecimento da necessidade de se proteger os ecossistemas e as áreas ameaçadas de degradação, porém não é feita nenhuma menção direta aos ecossistemas de áreas úmidas, sendo que esses princípios podem contribuir para a proteção desses sistemas.

Nota-se uma evolução do pensamento ambiental, principalmente devido a Conferência de Estocolmo no ano de 1972, cujo objetivo foi conscientizar o mundo sobre a necessidade do atendimento das necessidades da população de modo a não comprometer nossas gerações futuras.

Da mesma forma vaga, a Constituição da República Federativa de 1988 estabeleceu uma série de direitos (como um ambiente ecologicamente equilibrado, proteção do meio ambiente, combate à poluição) e trouxe alguns elementos passíveis de regulamentação (e.g. recursos hídricos, política urbana), além de dedicar um capítulo à matéria relativa ao meio ambiente. Ademais, é imprescindível destacar que o Art. 225 no seu quarto inciso enquadra a Floresta Amazônica brasileira, a Mata Atlântica, a Serra do Mar, o Pantanal Mato-Grossense e a Zona Costeira como patrimônio nacional, bem como assegura a preservação ambiental quanto ao uso dos recursos naturais, situação que destaca a importância das áreas úmidas (BRASIL, 1988).

Durante a década de noventa do século passado, importantes mecanismos normativos relacionados com as áreas úmidas foram aprovados. Durante o ano de 1992, o texto que foi fruto da Convenção Ramsar de 1972 foi adotado pelo Brasil em sua integra (Decreto Legislativo $\left.n^{\circ} 33 / 1992\right)$, contudo, sua promulgação somente ocorreu em 1996 (Decreto $n^{\circ}$ 1.905/1996) e assim, o governo brasileiro assumiu cumprir uma série de premissas. Dentre estas, pode-se citar: (i) a proteção das áreas úmidas; (ii) a compensação de perdas de área destes ecossistemas; (iii) o incentivo a condução de pesquisas científicas; e (iv) a formação de recursos humanos para o manejo adequado das áreas úmidas (BRASIL, 1992, 1996). 
Semelhantemente, outro acordo internacional ratificado pelo Brasil foi a Convenção sobre Diversidade Biológica (CDB), aprovado pelo Decreto Legislativo $n^{\circ}$ 2/1994 (BRASIL, 1994). Todavia, a promulgação deste decreto somente ocorreu em 1998 (Decreto Legislativo $n^{\circ}$ 2.519/1998). O preâmbulo da CDB tem como essência a conservação da biodiversidade, mas elementos relacionados acabam sendo abordados (e.g. proteção de áreas, elaboração de medidas de manejo, uso sustentável da biodiversidade, redução dos impactos negativos) (BRASIL, 1998).

Ainda nesta mesma década, foi aprovada a Política Nacional de Recursos Hídricos (Lei Federal 9.433/1997). Tal dispositivo trata a água como um recurso natural, assim como fornece bases para a realização do seu manejo mediante a consideração de fatores elencados explicitamente (e.g. ocupação da paisagem, promoção dos usos múltiplos da água, a gestão descentralizada, avaliação qualitativa e quantitativa dos recursos hídricos), contexto que pode ser aplicado as áreas úmidas (BRASIL, 1997).

A Resolução CONAMA n 341/2003 estabelece diretrizes que visam evitar o comprometimento de ecossistemas em decorrência do desenvolvimento de atividades ligadas ao turismo na zona costeira, dentre elas merece destaque a necessidade de não se comprometer às áreas que atuam como espaços de expansão dos manguezais, uma tipologia de área úmida (BRASIL, 2003). Já a Resolução CONAMA nº 357/05 dispõe sobre valores de referência para a manutenção da qualidade da água e traz bases para o monitoramento limnológico, podendo também favorecer a avaliação das áreas úmidas (BRASIL, 2005). Destaca-se que a Resolução CONAMA n $341 / 2003$ foi revogada, situação reconhecida pela Resolução CONAMA n488/18 (BRASIL, 2018).

Ainda dentro do contexto de áreas úmidas e considerando a CDB, merece destaque o documento com as Metas de Aichi, com horizonte até 2020 e fruto de uma conferência das partes realizada no Japão em 2010. De forma explícita, as metas envolvem: (i) redução dos impactos do uso dos recursos naturais; (ii) redução da degradação de ecossistemas naturais; (iii) adoção de limites ecológicos seguros e proteção de espécies; (iv) redução da poluição; (v) controle de espécies invasoras; (vi) proteção das águas interiores e regiões costeiras com vistas aos serviços ecossistêmicos; e (vii) a mitigação das mudanças climáticas; (viii) consideração do conhecimento popular e científico.

A LPVN (BRASIL, 2012) é o dispositivo responsável por dispor sobre critérios de proteção no que se refere à vegetação marginal dos corpos hídricos. Todavia, o mecanismo é marcado por retrocessos em relação ao mecanismo anteriormente vigente (Código Florestal de 1965 - Lei Federal, alterada pela Medida Provisória no 2.166-67/2001 e Lei o 7.803/1989), dado que ameaça a manutenção das áreas úmidas por reduzir a faixa de vegetação passível de ser protegida nos corpos hídricos e preconiza que sua mensuração deve ser mediante o leito regular do corpo hídrico, situação de desconsidera completamente às variações sazonais dos ambientes hídricos, além de não estabelecer uma faixa de proteção ao redor das áreas úmidas (BRASIL, 1965).

A Recomendação do Comitê Nacional de Áreas Úmidas (CNZU) n 7 de 11 de junho de 2015 trata sobre a definição das Áreas Úmidas Brasileiras e traz um sistema de classificação destes ecossistemas (BRASIL, 2015). Neste documento está presente a adoção de uma definição de áreas úmidas adaptada de Junk et al. (2014), orientações para a identificação da extensão de uma área úmida e o sistema de classificação que deve ser adotado (Figura 1). 
Fabio Leandro da Silva

Welber Senteio Smith

Áreas úmidas brasileiras: bases para o gerenciamento, serviços ecossistêmicos e estratégias de manejo

Figura1 - Classificação e delineamento das áreas úmidas brasileiras.

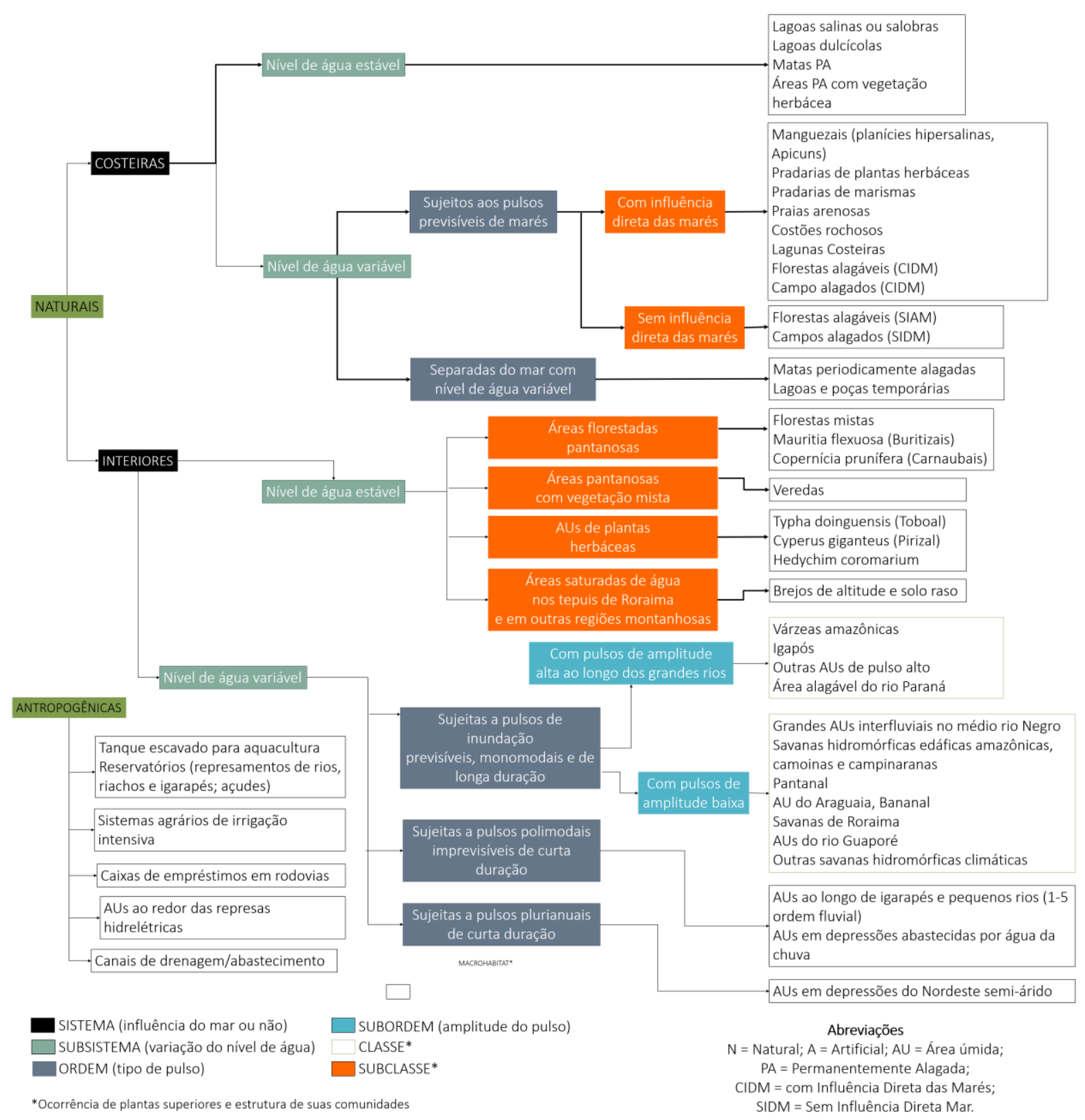

Fonte: Adaptado do Ministério do Meio Ambiente (2020) e Junk et al. (2014).

Um importante desdobramento da Convenção Ramsar é o Plano Estratégico Ramsar (2016-2024), que trata especificamente sobre os ecossistemas de áreas úmidas. Este acordo, estabelece 19 metas divididos em quatro objetivos estratégicos: (i) identificando os diretores de degradação e perda das áreas úmidas; (ii) conservação e manejo efetivo da Rede Ramsar; (iii) uso racional de todas as áreas úmidas; e (iv) favorecimento da implementação.

Um evento que ocorreu durante o primeiro semestre de 2019 foi a extinção do CNZU pelo poder executivo, organismo responsável por fornecer diretrizes e auxiliar na proposição de ações que visem o manejo adequado e conservação das áreas úmidas. Frente a liminar imposta pelo Supremo Tribunal Federal diante da necessidade de se cumprir as políticas públicas internacionais, o CNZU foi recriado por meio do Decreto $n^{\circ} 10.141 / 19$. Novamente, é ressaltado o caráter consultivo do órgão e o seu papel acerca de ações ligadas a Convenção Ramsar, todavia, a participação da sociedade civil foi reduzida. 


\section{SERVIÇOS ECOSSISTÊMICOS}

Serviços ecossistêmicos podem ser definidos basicamente como os benefícios que a sociedade obtém dos ecossistemas, sendo que esses serviços são agrupados em quatro categorias básicas: (i) provisão (e.g. fibras, água, madeira), (ii) regulação (e.g. doenças, qualidade da água), (iii) suporte (e.g. formação do solo, ciclagem de nutrientes) e (iv) culturais (e.g. estética, recreação) (MEA, 2005).

Os sistemas hídricos em seu estado natural fornecem uma ampla gama de serviços ecossistêmicos (GILVEAR; SPRAY; CASAS-MULET, 2013). Em relação aos ecossistemas de áreas úmidas, a sua contribuição para a melhoria da qualidade da água devido a remoção de nutrientes (e.g. fósforo, nitrogênio), a sua relação com a emissão de GEE e mudanças climáticas (HESSLEROVÁ et al. 2018; WIDNEY et al., 2018) são fatores amplamente reconhecidos. Watson et al. (2016) salientam que esses ecossistemas contribuem com o bem-estar humano devido a resiliência fornecida frente às mudanças climáticas. Os benefícios fornecidos pelas áreas úmidas desde o estabelecimento dos primeiros seres humanos nas áreas adjacentes a estes sistemas (KINGSFORD; BASSET; JACKSON, 2016).

Com base em Constanza et al. (1997), Burkhard et al. (2014) e Junk et al. (2014), os principais serviços ecossistêmicos fornecidos pelos ecossistemas de áreas úmidas são: (i) regulação do clima local; (ii) regulação de fluxo; (iii) purificação da água; (iv) regulação de nutrientes; (v) proteção natural contra alguns eventos extremos (e.g. inundações, enchentes); (vi) controle de pestes e doenças; (vii) diluição de efluentes; (viii) refúgios para a biodiversidade; (ix) regulação de GEE; (x) estoque de água; (xi) controle de processos erosivos e retenção de sedimentos; (xii) ciclagem de nutrientes; (xiii) provisão de alimentos para a biota e seres humanos; (xiv) recreação; (xv) oportunidades culturais; (xvi) recarga de aquíferos; (xvii) produção de madeira e produtos não madeireiros; (xix) plantas medicinais; (xx) pescado e (xxi) ecoturismo.

Apesar de serem responsáveis pelo fornecimento de uma grande quantidade de serviços ecossistêmicos, globalmente observa-se uma grande redução das áreas úmidas em decorrência das mudanças de uso da terra (SANNIGRAHI et al., 2018). Muitos serviços ecossistêmicos não podem ser valorados e a sua disponibilidade pode variar ao longo do tempo, situação que demanda o uso de métodos não monetários e a manutenção da integridade das áreas úmidas (SILVA et al., 2019b) para a continuidade da provisão. É importante enfatizar que a criação de áreas protegidas, identificação de áreas passíveis de restauração e restrições de uso (DOMÍNGUEZ-DOMÍNGUEZ et al., 2019) podem contribuir para este sentido.

\section{ESTRATÉGIAS DE MANEJO}

Um fator importante que pode contribuir para a restauração e até mesmo a conservação dos ecossistemas de áreas úmidas é o fato de esses processos podem ser economicamente atrativos para os usos agrícolas e urbanos, situação que favorece a manutenção dos serviços ecossistêmicos (BARTH e DÖLL, 2016). Sabe-se que uma abordagem com caráter estratégico pode contribuir para a conservação dos ecossistemas de áreas úmidas. A abordagem desenvolvida por Geneletti (2015), pautada na Avaliação Ambiental Estratégica e a provisão de serviços ecossistêmicos, pode contribuir neste sentido visto o potencial de influenciar ações estratégicas (e.g. planos, projetos e programas) e fornecer bases para a conservação das áreas úmidas frente aos impactos ambientais. Um exemplo pode ser visualizado na Figura 2. 
Figura 2 - Fluxograma de manejo das áreas úmidas pautada na Avaliação Estratégica

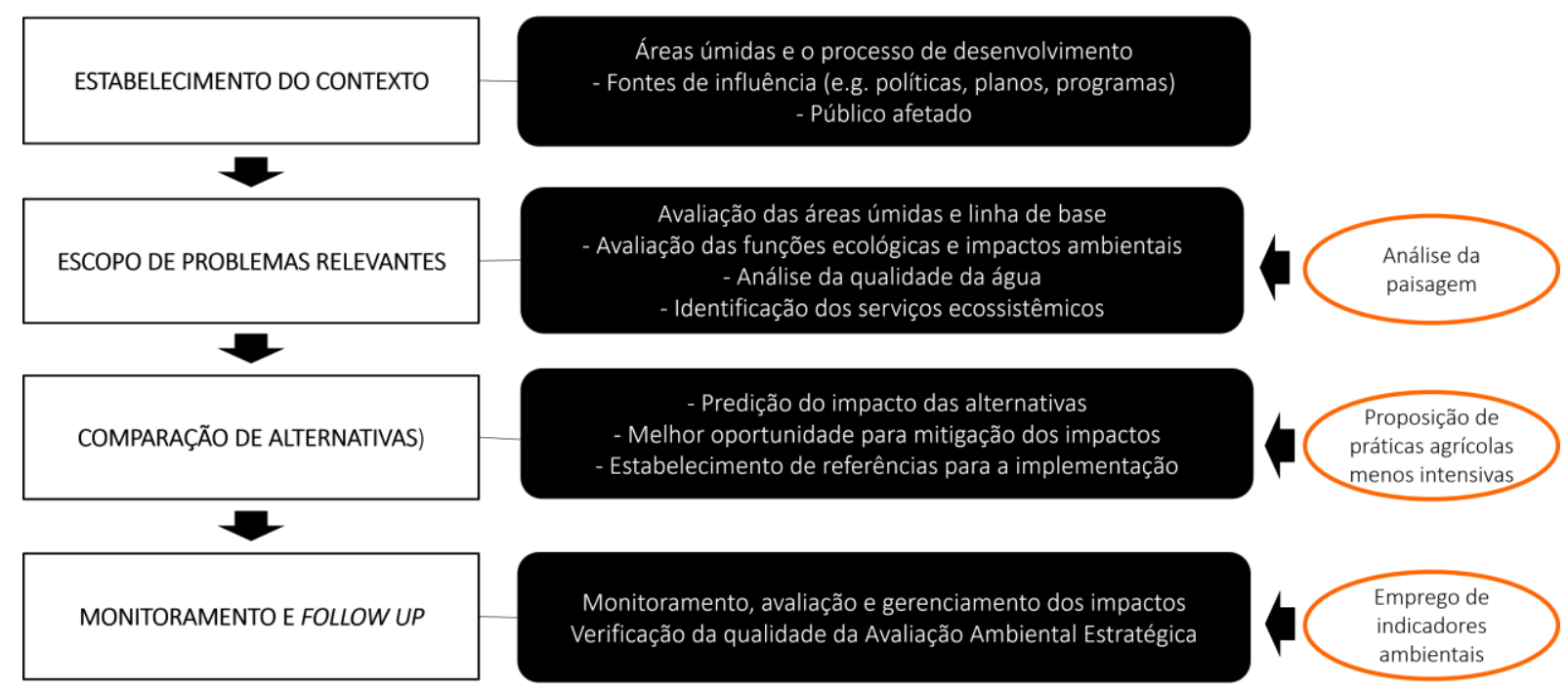

Fonte: Baseado em Therivel (2004), Geneletti (2015) e Silva et al. (2020b).

Ainda nesse contexto, reforça-se que apesar de existirem algumas divergências na determinação dos impactos cumulativos e impactos indiretos sobre as áreas úmidas conforme o método utilizado, uma abordagem ecológica dentro do processo de Avaliação de Impacto Ambiental (WAWRZYCZEK et al., 2018) pode contribuir para a conservação dos ecossistemas de áreas úmidas. $O$ emprego de indicadores ambientais (e.g. Vulnerabilidade da Paisagem, Fragilidade da Paisagem, Índice de Urbanidade) relacionados com o uso e cobertura da paisagem podem fomentar a tomada de decisão e contribui para a minimização dos impactos decorrentes do desenvolvimento das atividades humanas e planejamento da paisagem (e.g. SILVA et al., 2017).

A análise da paisagem voltada para as áreas úmidas favorece a verificação das taxas de conversão das áreas naturais em outros usos, assim como a dinâmica e extensão das áreas úmidas e sistemas correlacionados, proporcionando base para o manejo destes ambientes e auxiliando na proposição dos melhores cenários no que se refere a interferência humana (FUSHITA; SANTOS, 2017).

Destaca-se ainda que, uma análise da paisagem e seus padrões de mudança podem contribuir para o planejamento do território (SU et al., 2012). Tratando-se das áreas inseridas entre em um gradiente ruralurbano, a abordagem desenvolvida por Larondelle e Haase (2013) pode ser de grande valia, dado que se pauta nas alterações provocadas pelos usos e cobertura da terra e suas relações com os serviços ecossistêmicos prestados pelos ecossistemas ali existentes. Ainda no que refere-se à associação de padrões da paisagem e provisão de serviços ecossistêmicos, a abordagem desenvolvida por Burkhard, Müller e Windhorst (2009) e empregada por Burkhard et al. (2014) é capaz de refletir às demandas e capacidade de provisão dos serviços ecossistêmicos pelas áreas úmidas, fator que pode servir de base para a elaboração de estratégias de manejo no meio urbano.

Tratando-se das áreas úmidas inseridas na zona rural, a adoção de práticas agrícolas menos intensivas e pautadas em princípios ecológicos pode auxiliar na conservação desses sistemas além de salvaguardar sua biodiversidade associada. Mikulcal et al. (2013) salientam que políticas de biodiversidade e agricultura estão indissociavelmente relacionadas. Sendo assim, a formulação de políticas agrícolas que tragam o emprego de práticas menos impactantes do ponto de ecológico e que propiciem o desenvolvimento econômico, podem contribuir para a manutenção da integridade dos ecossistemas de áreas úmidas. Diante da dificuldade de se realizar o engajamento do poder público e a fraqueza das regulamentações de ordem ambiental frente às mudanças decorrentes do desenvolvimento regional, torna-se preciso necessária a associação entre diferentes escalas de interação sociológica (IORIS, 2013). Destaca-se que os esquemas de Pagamentos por Serviços Ambientais, ou seja, a provisão de incentivos econômicos para aqueles que contribuem para o gerenciamento ambiental (SCHULZ et al., 2015), pode favorecer a conservação dos ecossistemas de áreas úmidas nesses espaços. 
Fabio Leandro da Silva

Welber Senteio Smith

Áreas úmidas brasileiras: bases para o gerenciamento, serviços ecossistêmicos e estratégias de manejo

Marcela Bianchessi da Cunha-Santino Irineu Bianchini Junior

Lara-Pulido, Guevara-Sanguinés e Martelo (2018) ressaltam que a valoração dos serviços ecossistêmicos é uma ferramenta útil para a formulação de políticas. Entretanto, em alguns casos certos serviços (e.g. coprodutores) podem ser considerados como isentos de valor econômico, devido toda a complexidade envolvida (Figura 3), vários aspectos podem não ser considerados na valoração monetária, o que demanda a condução de outros métodos (SILVA et al., 2019b).

Figura 3 - Interfaces dos ecossistemas de áreas úmidas e sua complexidade associada com seus valores.

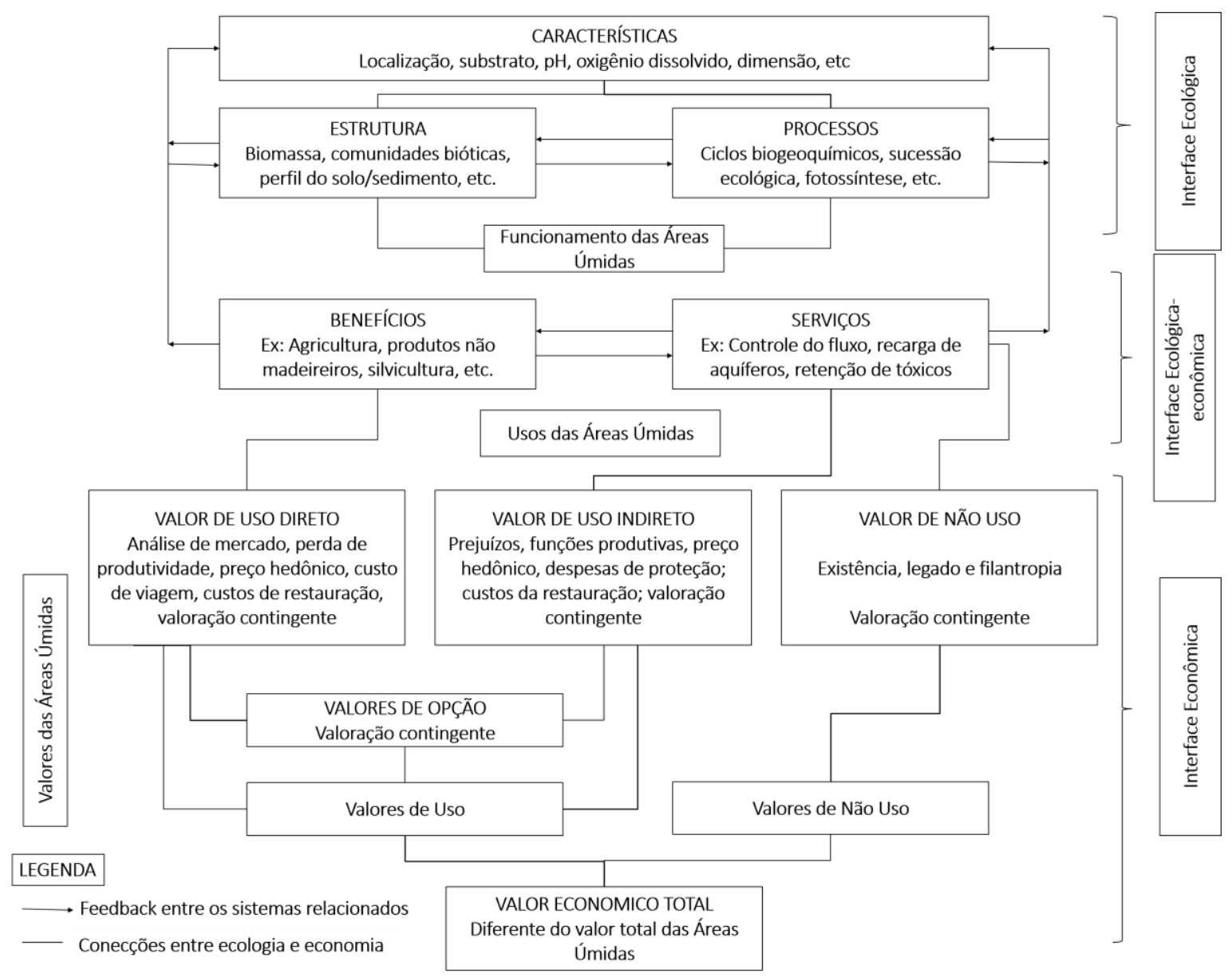

Fonte: Adaptado de Turner et al. (2000).

Diante do cenário de perda e/ou ruptura de conectividade das áreas úmidas, a restauração ambiental pode ser uma alternativa para a manutenção dos serviços ecossistêmicos prestados e reversão da desconexão hidrológica, através de projetos que busquem o emprego de estruturas verdes (e.g. parques lineares, áreas permeáveis) (GUIDA; REMO; SECCHI, 2016; WATSON et al., 2016) nas áreas urbanas e rurais. Hesslerová et al. (2018) destacam que o desmatamento e a drenagem de áreas úmidas situam-se entre os fatores mais significativos para as mudanças climáticas, além de que o emprego de espécies vegetais mais tolerantes ao calor e a falta de água pode ser uma medida a ser adotada em projetos de recuperação que visem reduzir a produção de GEE. Sendo assim, estratégias que visem a reabilitação ou restauração desses sistemas contribuem para à conservação ou recuperação desses ecossistemas. Contudo, é preciso compreender os processos que ocorrem nos corpos hídricos, nos sistemas socioecológicos, na escala espacial e possíveis restrições e que podem influenciar no processo (GILVEAR; SPRAY; CASAS-MULET et al., 2013) de recuperação e de decisão.

As áreas úmidas proporcionam uma série de benefícios para o bem-estar humano que possuem grande potencial econômico, é notório que a implementação de estratégias e ações que visem a restauração 
contribui para o aumento do valor destes serviços prestados, bem como assegura a sua manutenção (PERIOTTO; TUNDISI, 2018).

Basicamente, a restauração das áreas úmidas pode ser descrita como o retorno desses ecossistemas às suas condições anteriores ao distúrbio, entretanto, a restauração completa da funcionalidade destes sistemas pode não ser totalmente estabelecida (ZEDLER, 2000; SUCHARA, 2018). Tratando-se da vegetação marginal que circunda as áreas úmidas, os projetos de restauração necessitam selecionar uma área que sirva como referência para o pré-projeto, assim como sirva de base para o monitoramento do processo de restauração (HAUSNER et al., 2018).

Cabe salientar que os índices limnológicos podem ser de grande auxílio no monitoramento de projetos de restauração ou reabilitação, desde que exista uma área controle para comparações. Cvetkovic e ChowFraser (2011) salientam que os parâmetros limnológicos possuem forte associação com os usos e cobertura da terra. Os autores demostraram que o Water Quality Index, Wetland Macrophyte Index e Wetland Fish Index são ferramentas capazes de auxiliar no monitoramento e avaliação dos ecossistemas de áreas úmidas, bem como favorecem a formulação de possíveis estratégias de manejo e auxilia na verificação da eficácia de sua implementação. O emprego de macroinvertebrados bentônicos no processo de avaliação e monitoramento pode ser uma ótima estratégia, dado que tal comunidade responde aos distúrbios antropogênicos (e.g. BRAZNER et al., 2007; TANAKA et al., 2015). Por sua vez, a avaliação da qualidade do sedimento é um fator importante, uma vez que ele reflete a produtividade do ecossistema e pode acumular uma série de contaminantes de origem antropogênica, como os metais pesados e agrotóxicos (KULKARNI; DEOBAGKAR; ZINJARDE., 2018).

Ainda no que se refere à conservação da biodiversidade associada as áreas úmidas, frisa-se que, é indispensável a obtenção de informação genética para se identificar variações em populações e evitar a homogeneização, bem como propiciar a realização do monitoramento (e.g. CORRÊA et al., 2016). Em relação aos agroecossistemas, torna-se importante se avaliar possíveis estratégias que propiciem a conservação da biodiversidade nesses sistemas já alterados, principalmente na adoção de medidas ambientalmente sustentáveis (e.g. MACHADO; MALTCHIK, 2010). Nos casos onde existe contaminação, é preciso a realização do transporte biológico dos contaminantes, bem como a sua modulação pela comunidade biológica e o destino desses contaminantes nos ecossistemas (SCHIESARI; LEIBOLD; BURTON JÚNIOR., 2017). As práticas de manejo voltadas para a descontaminação dos fatores abióticos, geralmente envolvem o emprego da biorremediação, a fitorremediação (PEREIRA; FREITAS, 2012; COUTINHO et al., 2015) e técnicas oriundas da geotecnia, como contenções hidráulicas.

Por fim, destaca-se que uma política de caráter ambiental com objetivos bem definidos, medidas para a regulação e o estabelecimento de ações (VÉLEZ; GARCÍA; TENORIO, 2018) em nível nacional seria de grande auxílio para a proteção dos ecossistemas de áreas úmidas e proteção da biota associada frente ao cenário de mudanças climáticas. Dada a grande diversidade de tipologias existentes em relação a esses sistemas, as delimitações apresentadas por Gomes e Magalhães Júnior. (2017) ou Junk et al. (2014) para os atributos das áreas úmidas seria de grande valia para a elaboração de tal dispositivo normativo e o estabelecimento de atores responsáveis.

\section{CONSIDERAÇÕES FINAIS}

Os ecossistemas de áreas úmidas brasileiros carecem de um dispositivo normativo que trate sobre a sua proteção no âmbito nacional, apesar de existem outros mecanismos que podem fornecer bases para a sua proteção. Essas áreas representam cerca de um quinto do território nacional e são responsáveis pela provisão de diversos serviços ecossistêmicos. Dentre às principais estratégias para o seu adequado manejo, destacam-se aquelas medidas caracterizadas por terem um caráter mais estratégico e que se relacionam com o planejamento em curto, médio e longo prazo. Por sua vez, aquelas abordagem que consideram os componentes da paisagem e a capacidade de provisão de serviços ecossistêmicos são de grande auxílio, uma vez que fornecem bases para que ocorra a tomada de decisão, situação que pode ser útil para a priorização de áreas úmidas que devem passar por um processo de reabilitação ou até mesmo tentativas de restauração ecológica. Uma política nacional que contemplem os aspectos aqui destacados contribui para a efetivação das políticas públicas (inter)nacionais existentes e auxilia na proteção dos ecossistemas de áreas úmidas. Frente ao cenário de mudanças climáticas, torna-se necessário o monitoramento desses sistemas e adoção de medidas que propiciem a manutenção das espécies associadas, incluindo a sua persistência nesses ecossistemas em longo prazo. 


\section{AGRADECIMENTOS}

Os autores agradecem ao Conselho Nacional de Desenvolvimento Cientifico e Tecnológico (CNPq) pelo auxílio concedido (Processos: 830728/1999-6 e 158927/2018-4).

\section{REFERÊNCIAS}

BARTH, N. C.; DÖLL, P. Assessing the ecosystem service flood protection of a riparian forest by applying a cascade approach. Ecosystems Services, v. 21, p. 39-52, 2016. https://doi.org/10.1016/i.ecoser.2016.07.012

BOSSELMAN, F. Planning for a Bull Market for Wetlands. Planning \& Environmental Law: Issues and decisions that impact the built and natural environments, v. 61, n. 2, p. 3-14, 2009. https://doi.org/10.1080/15480750902743381

BOZELLI, R. L.; FARIAS, D. S.; LIMA, S. K. F.; LIRA, R. T. S.; NOVA, C. C.; SETUBAL, R. B.; SODRÉ, E. O. Pequenas áreas úmidas: importância para a conservação e gestão da biodiversidade brasileira. Diversidade e Gestão, v. 2, n. 2, p. 122 - 138, 2019.

BRASIL. Decreto $\mathrm{n}^{\circ} 24.643$, de 10 de julho de 1934. Disponível em: https://www2.camara.leg.br/legin/fed/decret/1930-1939/decreto-24643-10-julho-1934-498122publicacaooriginal-1-pe.html . Acesso em: 18 de agosto de 2020.

BRASIL. Lei $\mathrm{n}^{\circ} 4.771$, de 15 de setembro de 1965. Disponível em: http://www.planalto.gov.br/ccivil 03/leis//4771.htm . Acesso em: 18 de agosto de 2020.

BRASIL. Lei $\mathrm{n}^{\circ} 6.938$, de 31 de agosto de 1981. Disponível em: http://www.planalto.gov.br/ccivil 03/LEIS/L6938.htm . Acesso em: 18 de agosto de 2020.

BRASIL. Constituição da República Federativa do Brasil de 1988. Disponível em: http://www.planalto.gov.br/ccivil 03/constituicao/constituicaocompilado.htm . Acesso em: $18 \mathrm{de}$ agosto de 2020.

BRASIL. Decreto Legislativo $n^{\circ}$ 33, de 1992. Disponível em: https://www2.camara.leg.br/legin/fed/decleg/1992/decretolegislativo-33-16-junho-1992-358327publicacaooriginal-1-pl.html . Acesso em: 18 de agosto de 2020.

BRASIL. Decreto Legislativo $\mathrm{n}^{\circ} 2$, de 1994. Disponível em: https://www2.camara.leg.br/legin/fed/decleg/1994/decretolegislativo-2-3-fevereiro-1994-358280publicacaooriginal-1-pl.html . Acesso em: 18 de agosto de 2020.

BRASIL. Decreto $\mathrm{n}^{\circ} 1.905$, de 16 de maio de 1996. Disponível em: http://www.planalto.gov.br/ccivil 03/decreto/1996/D1905.htm . Acesso em: 18 de agosto de 2020.

BRASIL. Decreto $\mathrm{n}^{\circ} 2.519$, de 16 de março de 1998. Disponível em: http://www.planalto.gov.br/ccivil 03/decreto/D2519.htm . Acesso em: 18 de agosto de 2020.

BRASIL. Lei $n^{\circ}$ 9.433, de 8 de janeiro de 1997. Disponível em: http://www.planalto.gov.br/ccivil 03/LEIS/L9433.htm . Acesso em: 18 de agosto de 2020.

BRASIL. Resolução CONAMA $n^{\circ} 341$, de 25 de setembro de 2003. Disponível em: http://www2.mma.gov.br/port/conama/legiabre.cfm?codlegi=379. Acesso em: 18 de agosto de 2020.

BRASIL. Resolução CONAMA $n^{\circ} 357$, de 17 de março de 2005 . Disponível em: http://www2.mma.gov.br/port/conama/legiabre.cfm?codlegi=459. Acesso em: 18 de agosto de 2020.

BRASIL. Lei $\mathrm{n}^{\circ} 12.651$, de 25 de maio de 2012. Disponível em: http://www.planalto.gov.br/ccivil 03/ ato2011-

2014/2012/lei/l12651.htm\#: :text=1\%C2\%BA\%20(VETADO).-

,Art.,n\%C2\%BA\%20571\%2C\%20de\%202012) . Acesso em: 18 de agosto de 2020.

BRASIL. Recomendação CNZU $n^{\circ} 07$, de 11 de junho de 2015 . Disponível em: https://www.mma.gov.br/images/arquivo/80089/Recomendacao\%20CNZU\%20n\%207\%20conceito\% 20e\%20sistema\%20de\%20classificacao\%20de\%20areas\%20umidas\%201\%201.pdf . Acesso em: 18 de agosto de 2020.

BRASIL. Resolução CONAMA $n^{\circ} 488$, de 05 de setembro de 2018. Disponível em: 
http://www2.mma.gov.br/port/conama/legiabre.cfm?codlegi=737 . Acesso em: 18 de agosto de 2020.

BRAZNER J. C. et al. Responsiveness of Great Lakes Wetland Indicators to Human Disturbances at Multiple Spatial Scales: A Multi-Assemblage Assessment. Journal of Great Lakes Research, v. 33, n. 3, p. 42 - 66, 2007. https://doi.org/10.3394/0380-1330(2007)33[42:ROGLWI]2.0.CO;2

BURKHARD, B.; MÜLLER, F.; WINDHORST, W. Landscapes Capacities to Provide Ecosystem Services - a Concept for Land-Cover Based Assessments. Landscape Online, v. 15, p. 1 - 22, 2009. https://doi.org/10.3097/LO.200915

BURKHARD, B, KANDZIORA, M, HOU, Y, MÜLLER, F. Ecosystem Service Potential, Flows and Demands - Concept for spatial Localization, Indication and Quantification. Landscape Online, v. 34, p. 1 - 32, 2014. https://doi.org/10.3097/LO.201434

CHEN, Y.; SHIH, C. Sustainable management of coastal wetlands in Taiwan: a review for invasion, conservation, and removal of mangroves. Sustainability, v. 11, p. 1 - 16, 2019. https://doi.org/10.3390/su11164305

CORRÊA, T. C.; DEL LAMA, S. N.; SOUZA, J. R.; MIÑO, C. I. Genetic structuring among populations of the great egret, Ardea alba egretta, in major Brazilian wetlands. Aquatic Conservation Marine and Freshwater Ecosystems, v. 26, n. 2, p. 333-349, 2016. https://doi.org/10.1002/aqc.2588

COSTANZA, R. et al. The value of the world's ecosystem services and natural capital. Nature, v. 387, p. 253-260, 1997. https://doi.org/10.1038/387253a0

COUTINHO, P. W. R.; CADORIN, D. A.; NORETO, L. M.; GONÇALVES JÚNIOR., A. C. Alternativas de remediação e descontaminação de solos: biorremediação e fitorremediação. Nucleus, v. 12, n. 1, p. 59 - 68, 2015. https://doi.org/10.3738/1982.2278.1400

CVETKOVIC, M.; CHOW-FRASER, P. Use of ecological indicators to assess the quality of Great Lakes coastal wetlands. Ecological Indicators, v. 11, n. 6, p. 1609-1622, 2011. https://doi.org/10.1016/i.ecolind.2011.04.005

DIEGUES, A. S. The role of cultural diversity and communal participation in wetland management in Brazil. Landscape and Urban Planning, 20: 61 - 66, 1991. https://doi.org/10.1016/01692046(91)90092-Z

DOMÍNGUEZ-DOMÍNGUEZ, M.; ZAVALA-CRUZ, J.; RINCÓN-RAMÍREZ, J. A.; MARTíNEZZURIMENDI, P. Management strategies for the conservation, restoration and utilization of mangroves in Southeastern Mexico. Wetlands, v. 39, p. 907 - 919, 2019. https://doi.org/10.1007/s13157-01901136-z

FUSHITA, A. T.; SANTOS, J. E. Land use change trajectories for wetland management (Mogi-Guaçu Floodplain River, Southern Brazil). Journal of Geoscience and Environmental Protection, v. 5, p. 6276, 2017. https://doi.org/10.4236/gep.2017.510006

GENELETTI, D. A. Conceptual Approach to Promote the Integration of Ecosystem Services in Strategic Environmental Assessment. Journal of Environmental Assessment Policy and Management, v. 17, p. 1 - 27, 2015. https://doi.org/10.1142/S1464333215500350

GILVEAR, D. J.; SPRAY, C. J.; CASAS-MULET, R. River rehabilitation for the delivery of multiple ecosystem services at the river network scale. Journal of Environmental Management, v. 126, p. 30 43, 2013. https://doi.org/10.1016/i.jenvman.2013.03.026

GOMES, C. S.; MAGALHÃES JÚNIOR, A. P. Aparato conceitual sobre áreas úmidas (wetlands) no Brasil: desafios e opiniões de especialistas. Boletim Goiano de Geografia, v. 37, n. 3, p. 484 - 508, 2017. https://doi.org/10.5216/bgg.v37i3.50767

GONÇALVES, L. M.; TSUGE, M. T.; BORGHI, V. S.; MIRANDA, F. P.; SALES, A. P. A.; LUCCHETTI, A. L. G.; LUCCHETTI, G. Spirituality, religiosity, quality of life and mental health among Pantaneiros: a study involving a vulnerable population in Pantanal Wetlands, Brazil. Journal of Religion \& Health, v. 57, p. 2431-2443, 2018. https://doi.org/10.1007/s10943-018-0681-4

GRASEL, D; MORMUL, R. P.; BOZELLI, R. L., THOMAZ, S. M., JARENKOW, J. A. Brazil's Native Vegetation Protection Law threatens to collapse pound functions. Perspectives in Ecology and Conservation, v. 16, p. 234 - 237, 2018. https://doi.org/10.1016/j.pecon.2018.08.003

GUIDA, R. J.; REMO, J. W. F.; SECCHI, S. Tradeoffs of strategically reconnecting rivers to their 
floodplains: The case of the Lower Illinois River (USA). Science of The Total Environment, v. 572, p. 43 - 55, 2016. https://doi.org/10.1016/i.scitotenv.2016.07.190

HAUSNER, M. B.; HUNTINGTON, J. L.; NASH, C.; MORTON, D.; MCEVOY, D. J.; PILLIOD, D. S.; HEGEWISCH, K. C.; DAUDERT, B.; ABATZOGLOU, J. T.; GRANT, G. Assessing the effectiveness of riparian restoration projects using Landsat and precipitation data from the cloud-computing application

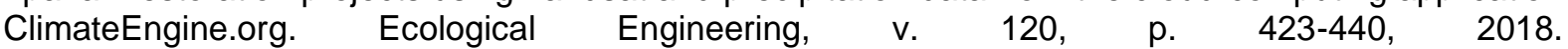
https://doi.org/10.1016/i.ecoleng.2018.06.024

HESSLEROVÁ, P; HURYNA, H; POKORNÝA, J; PROCHÁZKAB, J. The effect of forest disturbance on landscape temperature. Ecological Engineering, v. 120, p. 345-354, 2018. https://doi.org/10.1016/i.ecoleng.2018.06.011

IORIS, A. A. R. Rethinking Brazil's Pantanal Wetland Beyond Narrow Development and Conservation Debates. The Journal of Environment \& Development, v. 22, n. 3, p. 239-260, 2013. https://doi.org/10.1177/1070496513493276

JUNK, W. J. Current state of knowledge regarding South America wetlands and their future under global climate change. Aquatic Sciences, v. 75, p. 113 - 131, 2013. https://doi.org/10.1007/s00027012-0253-8

JUNK, W. J.; PIEDADE, M. T. F. Áreas Úmidas (AUs) Brasileiras: Avanços e Conquistas Recentes. Boletim ABLimno, v. 41, n. 2, p. 20 - 24, 2015.

JUNK, W. J.; PIEDADE, M. T. F.; LOURIVAL, R.; WITTMANN, F.; KANDUS, P.; LACERDA, L. D.; BOZELLI, R. L.; ESTEVES, F. A.; CUNHA, C. N.; MALTCHIK, L.; SCHÖNGART, J.; SCHAEFFER-NOVELLI,Y., AGOSTINHO, A. A. Brazilian wetlands: their definition, delineation, and classification for research, sustainable management, and protection. Aquatic Conservation: Marine and Freshwater Ecosystems, v. 24, n. 1, p. 5-22, 2014. https://doi.org/10.1002/aqc.2386

KINGSFORD, R. T.; BASSET, A.; JACKSON, L. Wetlands: conservation's poor cousins. Aquatic conservation: marine and freshwater ecosystems, v. 26, p. 892 - 916, 2016. https://doi.org/10.1002/aqc.2709

KULKARNI, R.; DEOBAGKAR, D.; ZINJARDE, S. Metals in mangrove ecosystems and associated biota: A global perspective. Ecotoxicology and Environmental Safety, v. 153, p. 215-228, 2018. https://doi.org/10.1016/j.ecoenv.2018.02.021

LARA-PULIDO, J. A.; GUEVARA-SANGUINÉS, A.; MARTELO, C. A. A meta-analysis of economic valuation of ecosystem services in Mexico. Ecosystem Services, 31: 126-141, 2018. https://doi.org/10.1016/j.ecoser.2018.02.018

LARONDELLE, N.; HAASE, D. Urban ecosystem services assessment along a rural-urban gradient: A cross-analysis of European cities. Ecological Indicators, 29: 179 - 190, 2013. https://doi.org/10.1016/j.ecolind.2012.12.022

LIPCZYNSKI-KOCHANY, E. Effect of climate change on humic substances and associated impacts on the quality of surface water and groundwater: A review. Science of The Total Environment, v. 640641, p. 1548-1565, 2018. https://doi.org/10.1016/j.scitotenv.2018.05.376

MACHADO, I. F.; MALTCHIK, L. Can management practices in rice fields contribute to amphibian conservation in southern Brazilian wetlands?. Aquatic Conservation: Marine and Freshwater Ecosystems, v. 20, n. 1, p. 39-46, 2010. https://doi.org/10.1002/aqc.1070

MALTCHIK, L.; CALEFFI, V.; STENERT, C.; BATZER, D. P.; PIEDADE, M. T. F.; JUNK, W. J. Legislation for wetland conservation in Brazil: Are existing terms and definitions sufficient? Environmental Conservation, v. 45, n. 3, p. $301 \quad$ - $305,2018$. https://doi.org/10.1017/S0376892917000522

MEA - MILLENNIUM ECOSYSTEM ASSESSMENT. Ecosystems and Human Wellbeing: Synthesis. Washington, DC: Island Press, 2005, 137 pp.

MINISTÉRIO DO MEIO AMBIENTE - MMA. Áreas Úmidas - Convenção de Ramsar. 2020. Disponível em: https://www.mma.gov.br/biodiversidade/biodiversidade-aquatica/zonas-umidas-convencao-deramsar.html . Acesso em: 11 de março de 2020. 
MIKULCAL, F.; NEWING, J.; MILCU, A. I.; HARTEL, T. Integrating rural development and biodiversity conservation in Central Romania. Environmental Conservation, v. 40, n. 2, p. 129 - 137, 2013. https://doi.org/10.1017/S0376892912000392

OMERNIK, J. M.; GRIFFITH, G. E. Ecoregions of the Conterminous United States: Evolution of a Hierarchical Spatial Framework. Environmental Management, 54(6): 1249-1266, 2014. https://doi.org/10.1007/s00267-014-0364-1

PEREIRA, A. R. B.; FREITAS, D. A. F. Uso de micro-organismos para a biorremediação de ambientes impactados. Revista Eletrônica em Gestão, Educação e Tecnologia Ambiental, v. 6, n. 6, p. 995 - 1006, 2012. https://doi.org/10.5902/223611704818

PERIOTTO, N. A.; TUNDISI, J. G. A characterization of ecosystem services, drivers and values of two watersheds in São Paulo State, Brazil. Brazilian Journal of Biology, v. 78, n. 3, p. 397-407, 2018. https://doi.org/10.1590/1519-6984.166333

PIYAPONG, J.; RIRUENGRONG, R.; WIPAWEE, I.; SIRIPHAN, N.; PASSANAN, A. Empirical evidence of the roles of public spaces and public activities in the promotion of community relations and sense of place in a coastal community. Journal of Place Management and Development, v. 12, n. 4, p. 485-507, 2019. https://doi.org/10.1108/JPMD-09-2018-0065

POLAZ, C. N. M.; FERREIRA, F. C.; PETRERE JÚNIOR., M. The protected areas systems in Brazil as a baseline condition for wetlands management and fish conservancy: the example of the Pantanal National Park. Neotropical Ichthyology, v. 15, n. 3, e170041, 2017. https://doi.org/10.1590/1982-0224$\underline{20170041}$

ROITMAN, I.; VIEIRA, L. C. G.; JACOBSON, T. K. B.; BUSTAMANTE, M. M. C.; MARCONDES, N. J. S.; CURY, K.; ESTEVAM, L. S.; RIBEIRO, R. J. C.; RIBEIRO, V.; STABILE, M. C. C.; MIRANDA FILHO, R. J.; AVILA, M. L. Rural Environmental Reinnovative model for land-use and environmental policies. Land Use Policy, v. 76, p. 95 - 102, 2018. https://doi.org/10.1016/j.landusepol.2018.04.037

SANNIGRAHI, S.; BHATT, S.; RAHMAT, S.; PAUL, S. K.; SEN, S. Estimating global ecosystem service values and its response to land surface dynamics during 1995-2015. Journal of Environmental Management, v. 233, p. 115 - 131, 2018. https://doi.org/10.1016/j.jenvman.2018.05.091

SCHIESARI, L.; LEIBOLD, M. A.; BURTON JÚNIOR, G. A. Metacommunities, metaecosystems and the environmental fate of chemical contaminants. Journal of Applied Ecology, v. 55, n. 3, p. 15531563, 2017. https://doi.org/10.1111/1365-2664.13054

SCHULZ, C.; IORIS, A. R.; MARTIN-ORTEGA, J.; GLENK, K. Prospects for Payments for Ecosystem Services in the Brazilian Pantanal: A Scenario Analysis. Journal of Environmental \& Development, v. 24, n. 1, p. 26 - 53, 2015. https://doi.org/10.1177/1070496514548580

SILVA, F. L.; OLIVEIRA, E. Z.; PICHARILLO, C.; RUGGIERO, M. H.; COSTA, W. C.; MOSCHINI, L. E. Naturalidade da paisagem verificada por meio de indicadores ambientais: manancial do Rio Monjolinho, São Carlos-SP. Revista Brasileira de Geografia Física, v. 10, n. 3, 970 - 980, 2017. https://doi.org/10.5935/1984-2295.20170063

SILVA, F. L.; STEFANI, M. S.; SMITH, W. S.; CUNHA-SANTINO, M. B.; BIANCHINI JÚNIOR., I (a). The municipality role in Brazilian wetlands conservation: the establishment of connections among the Master Plan, the National Hydric Resources Policy and two international strategic plans. Revista Brasileira de Geografia Física, v. 12, n. 6, p. 2193 - 2203, 2019. https://doi.org/10.26848/rbgf.v12.6.p2193-2203

SILVA, F. L.; SMITH, W. S.; CUNHA-SANTINO, M. B.; BIANCHINI JÚNIOR.; I (b). Método não monetário para a avaliação dos serviços ecossistêmicos prestados pelas áreas úmidas em áreas urbanas. Revista Brasileira de Geografia Física, v. 12, n. 1, p. 01 - 08, 2019. https://doi.org/10.26848/rbgf.v12.1.p001-008

SILVA, F. L.; STEFANI, M. S.; SMITH, W. S.; SCHIAVONE, D. C.; CUNHA-SANTINO, M. B.; BIANCHINI JÚNIOR., I. (a) An applied approach for the assessment of anthropogenic disturbances in urban wetlands and the contributor river. Ecological Complexity, v. 43, p. 1 - 12, 2020. https://doi.org/10.1016/j.ecocom.2020.100852

SILVA, F.L.; STEFANI, M. S.; SMITH, W. S.; CUNHA-SANTINO, M. B.; BIANCHINI JÚNIOR.; I. (b). A conceptual framework to promote wetlands ecosystems management in strategic environmental 
assessment. Revista Brasileira de Geografia Física, v. 13, n. 5, 2020 . No prelo. https://doi.org/10.26848/rbgf.v13.5.p2114-2127

SMITH W. S.; SILVA F. L.; AMORIM S. R.; STEFANI, M. S. Urban biodiversity: how the city can do its management?. Biodiversity International Journal, v. 2, n. 3, p. 246-251, 2018. https://doi.org/10.15406/bii.2018.02.00068

SU, S.; XIAO, R.; JIANG, Z.; ZHANG, Y. Characterizing landscape pattern and ecosystem service value changes for urbanization impacts at an eco-regional scale. Applied Geography, v. 34, p. 295 305, 2012. https://doi.org/10.1016/j.apgeog.2011.12.001

SUCHARA, I. The impact of floods on the structure and functional processes of floodplain ecosystems. Journal of Soil and Plant Biology, v. 1, p. 44 - 60, 2018.

TANAKA, M. O.; SOUZA, A. L. T.; OLIVEIRA, A. K.; MOSCHINI, L. E. Redundância entre métricas da qualidade ambiental de riachos em paisagem agrícola. Revista Ambiente \& Água, v. 10, n. 4, p. 832 846, 2015. https://doi.org/10.4136/ambi-agua. 1665

THERIVEL, R. Strategic Environmental Assessment in Action. $1^{\circ}$ ed. Londres: Earthscan, 2004, 269 p.

TUNDISI, J. G.; MATSUMURA-TUNDISI, T. Impactos potenciais das alterações do Código Florestal nos recursos hídricos. Biota Neotropica, v. 10, n. 4, p. 67-75, 2010. https://doi.org/10.1590/S1676$\underline{06032010000400010}$

TURNER, R. K.; VAN DEN BERGH, J. C. J. M.; SÖDERQVIST, T.; BARENDREGT, A.; VAN DER STRAATEN, J.; MALTBY, E.; VAN IERLAND, E. C. Ecological-economic analysis of wetlands: scientific integration for management and policy. Ecological Economics, v. 35, p. 7 - 23, 2000. https://doi.org/10.1016/S0921-8009(00)00164-6

VÉLEZ, J. M. M.; GARCÍA, S. B.; TENORIO, A. E. Policies in coastal wetlands: Key challenges. Environmental Science \& Policy, v. 88, p. 72-82, 2018. https://doi.org/10.1016/i.envsci.2018.06.016

WATSON, K. B.; RICKETTS, T.; GALFORD, G.; POLASKY, S.; O'NIEL-DUNNEC, J. Quantifying flood mitigation services: The economic value of Otter Creek wetlands and floodplains to Middlebury, VT. Ecological Economics, v. 130, p. 16-24, 2016. https://doi.org/10.1016/.ecolecon.2016.05.015

WAWRZYCZEK, J; LINDSAY, R; METZGER, MJ; QUÉTIERC, F. The ecosystem approach in ecological impact assessment: Lessons learned from windfarm developments on peatlands in Scotland. Environmental Impact Assessment Review, v. 72, p. 157-165, 2018. https://doi.org/10.1016/i.eiar.2018.05.011

WIDNEY, S.; KANABROCKI KLEIN, A.; EHMAN, J.; HACKNEY, C.; CRAFT, C. The value of wetlands for water quality improvement: an example from St. Johns River watershed, Florida. Wetlands Ecology and Management, v. 26, p. 265 - 276, 2018. https://doi.org/10.1007/s11273-0179569-4

YANG, W.; JIN, Y.; SUN, T.; ZHIFENG, Y.; CAI, Y.; YI, Y. Trade-offs among ecosystem services in coastal wetlands under the effects of reclamation activities. Ecological Indicators, v. 92, p. 354 - 366, 2018. https://doi.org/10.1016/i.ecolind.2017.05.005

XAVIER, R. A.; CAMARGO, V. C.; CARDOSO, O. R.; TASSI, R. Eco-hidrologia integrada ao manejo dos recursos hídricos em áreas úmidas: caso do Banhado do Taim, RS. Engenharia Sanitaria e Ambiental, v. 24, n. 1, p. 187-197, 2019. https://doi.org/10.1590/s1413-41522019147356

ZALEWSKI, M. Ecohydrology for compensation of Global Change. Brazilian Journal of Biology, v. 70, n. 3, p. 689-695, 2010. https://doi.org/10.1590/S1519-69842010000400001

ZEDLER, J. B. Progress in wetland restoration ecology. Trends in Ecology \& Evolution, v. 15, n. 10, p. 402 - 407, 2000. https://doi.org/10.1016/S0169-5347(00)01959-5

Recebido em: 31/03/2020

Aceito para publicação em: 13/08/2020 